\section{Kinase convergence on elF4F}

\section{By Kai-Jye Lou, Senior Writer}

Researchers in France have shown that resistance to BRAF and MEK inhibitors in melanoma and possibly other cancers can be driven by pathways that converge on the eukaryotic translation initiation factor $4 \mathrm{~F}$ complex. ${ }^{1}$ The findings suggest developing inhibitors of the complex in combination with marketed kinase inhibitor drugs could help circumvent resistance.

For the few biotechs already developing cancer compounds that disrupt eukaryotic translation initiation factor $4 \mathrm{~F}$ (eIF4F), such as Egenix Inc. and Isis Pharmaceuticals Inc., the reported data suggest their candidates could have broader utility than previously realized.

eIF4F is a key complex that enables mRNA translation. It is comprised of the eIF4A RNA helicase, the eIF4E cap-binding protein and the eIF4G scaffolding protein. eIF4E binding to eIF $4 \mathrm{G}$ promotes the assembly of the eIF $4 \mathrm{~F}$ complex, which leads to increased mRNA translation. All three components of the complex have previously been linked to the development or progression of various cancers, and disruption of their activity has been shown to have anticancer effects. ${ }^{2,3}$

Egenix is developing small molecules for cancer that inhibit the interaction between eIF4E and eIF4G. The biotech plans to select its lead candidate within a year. Isis' antisense oligonucleotide ISISEIF4ERx targets eIF4E and is in Phase I/II testing to treat non-small cell lung cancer (NSCLC) and castration-resistant prostate cancer (CRPC).

Now, a group co-led by Stéphan Vagner has published data in Nature showing that the eIF4F complex also acts as a nexus for resistance to BRAF and MEK inhibitors, which are marketed for melanoma but are also being developed to treat a broad range of other cancers. Vagner is research director at the Institut National de la Santé et de la Recherche Médicale (INSERM).

Patients who initially respond to BRAF or MEK inhibitors invariably develop resistance. eIF4F acts downstream of the resistance mechanisms identified for BRAF and MEK inhibitors, such as those involving the MAPK and phosphoinositide 3-kinase (PI3K) pathways. ${ }^{4}$ Several compounds targeting those pathways are now entering the clinic in combination with BRAF and MEK inhibitors.

Vagner wanted to know whether there could be a common downstream target of MAPK and PI3K pathways that might be exploited to overcome resistance. He thought persistent formation of the eIF4F complex caused by increased PI3K and MAPK signaling could be driving resistance to BRAF and MEK inhibitors in melanoma.
"We had the feeling that most of the work that was published on the mechanisms underlying resistance to BRAF and MEK inhibitors somehow overlooked what lies downstream of ERK in the MAPK pathway or mTOR in the PI3K pathway," he said. "We therefore addressed the potential role of the eIF4F eukaryotic translation initiation complex, which regulates the initiation of the cap-dependent mRNA translation and whose formation is regulated downstream of ERK and mTOR."

\section{Complex experiments}

Vagner's team found that in drug-sensitive melanoma cell lines, the marketed BRAF inhibitors Zelboraf vemurafenib and Tafinlar dabrafenib, as well as the marketed MEK inhibitor Mekinist trametinib, caused changes in the interactions of eIF4E and eIF4G and led to decreased formation of the eIF $4 \mathrm{~F}$ complex compared with vehicle. By contrast, in drug-resistant human melanoma cell lines and mouse xenograft models, MEK and BRAF inhibitors did not decrease eIF4F formation compared with vehicle.

Roche, Chugai Pharmaceutical Co. Ltd. and Daiichi Sankyo Co. Ltd. market Zelboraf to treat unresectable or metastatic melanomas. GlaxoSmithKline plc markets Tafinlar and Mekinist as monotherapies in the U.S. and EU for the same indication. The pharma also markets the two drugs as a combination therapy for the indication in the U.S.

Next, the team tested whether targeting the various components of the eIF4F complex could resensitize cells to BRAF or MEK inhibitors. In the drug-resistant melanoma cell lines, Zelboraf combined with an inhibitor of the eIF4E-eIF4G interaction or inhibitors of eIF4A synergistically decreased tumor growth. In a mouse xenograft model of drug-resistant melanoma, Zelboraf plus an eIF4A inhibitor decreased tumor growth compared with the eIF4A inhibitor alone or vehicle.

Finally, the team showed that eIF4F complex formation was greater than baseline in biopsy samples from seven patients with melanoma whose tumors progressed on Zelboraf or Tafinlar. The biopsies were taken before or during treatment, and all tumors that progressed showed the effect.

Researchers at the Gustave Roussy Institute, University of Paris-Sud 11 and University of Strasbourg contributed to the study in addition to the INSERM group.

"The reported data suggest that drug developers might not need to come up with new anti-BRAF or anti-MEK molecules to go after mutated forms of the protein that resist current inhibitors," said Carston Wagner, a professor in the Department of Medicinal Chemistry and director of the Chemical Biology Initiative at the University of Minnesota College of Pharmacy. "Instead, they might be able to use their current inhibitors in combination with, say, a 4E-targeted therapy to address resistance."

Vagner suggested a viable clinical strategy might be to treat patients with a triple blockade consisting of anti-BRAF, anti-MEK and anti-eIF4F therapies, or to initiate treatment with anti-BRAF plus anti-MEK therapies and then add an eIF4F blocker when resistance develops.

\section{Not your typical class}

Jedd Levine, CMO of Egenix, told SciBX that the data reported by Vagner's group open up new therapeutic opportunities for compounds targeting members of the eIF $4 \mathrm{~F}$ complex. 


\section{Box 1. elF4A profiled.}

\section{A team at the Memorial Sloan-}

Kettering Cancer Center has published data in Nature showing that inhibition of the RNA helicase eukaryotic translation initiation factor $4 \mathrm{~A}$ (elF4A) could preferentially block the translation of multiple oncogenes. ${ }^{8}$

The researchers set out to profile the activity of elF4A in T cell acute lymphoblastic leukemia (T-ALL) because the molecular mechanisms underlying the anticancer effects of elF4A inhibitors such as silvestrol were unclear.

In a mouse model of T-ALL, vectorinduced overexpression of EIF4A1 accelerated leukemia development compared with a control vector. In T-ALL cells from patient samples and mice, the naturally derived elF4FA inhibitor silvestrol induced cell death with nanomolar $\mathrm{IC}_{50}$ values. In a mouse xenograft model of T-ALL, i.p. injection of silvestrol or a synthetic analog delayed tumor growth, whereas vehicle did not.

Next, the researchers used ribosome footprinting to analyze the effects of silvestrol-mediated elF4A inhibition on mRNA translation in a human T-ALL cell line. The analysis showed strong enrichment of a 12-nucleotide (CGG) motif and related 9-nucleotide motifs in the group of mRNAs encoded by genes downregulated by silvestrol $\left(p=2.2 \times 10^{-16}\right)$, suggesting such motifs mark mRNAs that depend on elF4A for translation. The researchers found that these motifs coincided with computationally predicted G-quadruplex structures in mRNA.

The group of genes encoding these elF4A-dependent mRNAs included many well-known oncogenes, such as $B$ cell lymphoma 2 (BCL-2; BCL2), c-Myc (MYC) and notch 1 (NOTCH1). Moreover, the mRNAs encoded by other well-known cancer-associated genes such as neuroblastoma Ras viral (v-Ras) oncogene (NRAS) and VEGF are known to contain G-quadruplex structures. ${ }^{10,11}$

Importantly, silvestrol did not downregulate housekeeping genes, and the naturally derived product and its synthetic analog were both well tolerated in mice.

"We found that inhibiting the 4A RNA helicase does not inhibit all mRNA translation and instead only targets a subset of mRNAs with this specific G-quadruplex structure," said Hans-Guido Wendel, the co-corresponding author and an associate member of the Cancer Biology \& Genetics Program at MSKCC. "We found that this G-quadruplex structure is highly conserved across organisms and found in lineage-restricted transcription factors, including in many oncogenes."

$\mathrm{He}$ added that blocking the translation of G-quadruplex-containing mRNAs through elF4A represents a new way to inhibit the production of multiple oncogenes that have traditionally been viewed as undruggable, such as $c-M y c$.

Wendel said that the key challenges going forward will be to obtain enough silvestrol to carry out the necessary studies to characterize its pharmacological properties and develop elF4A inhibitors that could be protected by IP. He noted that the global supply of silvestrol is on the order of milligrams per year and that current synthetic analogs "are very difficult to synthesize."

However, Wendel noted that a large drug developer should have the capabilities needed to synthesize sufficient quantities of such analogs for preclinical and clinical studies. He added that his group is interested in collaborating to move such projects forward.

MSKCC has filed a patent application covering assays that could be used to screen for compounds that work via the mechanism described in the Nature paper. The technology is available for licensing.
"Vagner's work really supports the idea that eIF4E, and more broadly the eIF4F complex, is a convergence point for resistance to targeted cancer therapies," said Levine. "The data suggest development of compounds to circumvent resistance that target upstream of the complex may not be as fruitful as pursuing compounds that target the complex itself."

However, although at least two companies are developing programs targeting eIF4F, a major challenge to drug discovery is that none of the components of the complex comes from protein classes that are easily druggable.

"Targeting the eIF4F complex is more complicated than targeting a kinase. The binding of $4 \mathrm{E}$ to $4 \mathrm{G}$, for example, is a protein-protein interaction, which is considered difficult to drug with small molecules," said Levine.

Wagner agreed. "Traditional high throughput screening efforts to discover small molecule inhibitors against eIF4E have not fared well," he said. However, he noted that eIF4E still represents one of the most promising targets within the complex as it has a deep binding pocket that makes it particularly amenable to rational drug design strategies.

Wagner also thinks that cancer cells are less likely to develop resistance to compounds targeting the eIF4F complex because the genes and proteins of the complex are highly conserved and important to fundamental cellular functions. "Mutations in these highly conserved regions are more likely to have detrimental effects on the organism," he said.

Vagner said that his group is now testing several new, undisclosed potential inhibitors against the eIF4F complex to select a lead candidate for the clinic. He said his group is interested in collaborating with a drug developer and/or licensing compounds to accelerate their preclinical and clinical development.

Egenix is addressing the challenge of drugging the eIF4E-eIF4G protein-protein interaction by developing small molecules that target an allosteric site on eIF4E. Levine said that targeting this site alters the protein's conformation in a manner that prevents its interaction with eIF4G.

\section{Indexing safety}

Given its role in regulating a process as fundamental as mRNA translation, another concern with targeting the eIF4F complex is whether compounds can be created with an acceptable therapeutic index. 
An alternative strategy for disrupting the eIF4F complex could be to inhibit the eIF4A RNA helicase.

At least three naturally derived products-silvestrol, hippuristanol and pateamine $\mathrm{A}$-are known to inhibit the RNA helicase eIF4A. However, these molecules are research compounds and generally lack the necessary ADME properties to be advanced as therapeutic candidates. ${ }^{5,6}$ Wagner noted that optimization of eIF4A inhibitors' pharmacological profiles has been difficult. In addition, he said, they are not as specific as the available eIF4E-targeted compounds.

But Hans-Guido Wendel was more optimistic about eIF4A inhibitors. "One might be concerned that targeting the translational initiation complex would be very toxic, but we have been able to deliver silvestrol at doses that were effective and well tolerated in animal models," he said. "We've found that silvestrol has potent anticancer effects at 0.2 and $0.5 \mathrm{mg} / \mathrm{kg}$, and drug developers are usually happy if their drugs work at $100 \mathrm{mg} / \mathrm{kg}$. The main toxicities we've seen are hematopoietic in origin, but this is common with many cancer therapies."

Wendel is an associate member of the Cancer Biology \& Genetics Program at Memorial Sloan-Kettering Cancer Center.

In 2011, Wendel's group showed that silvestrol can effectively block oncogenic survival signals in mouse models of lymphoma with no overt toxicity. ${ }^{7}$ His group recently published a study that describes eIF4A inhibition as a new strategy to block multiple well-known oncogenes (see Box 1, "eIF4A profiled"). ${ }^{8}$

Levine believes targeting eIF4E can be safely done. He noted that the published Phase I data for ISIS-EIF4ERx suggest that inhibiting eIF4E is well tolerated in patients because $87 \%$ of those treated with ISIS-EIF4ERx experienced only grade 1 or 2 toxicities. ${ }^{9}$ He added that Egenix also detected no significant toxicity in its animal safety studies with a small molecule inhibitor of eIF4E-eIF4G.

Isis declined requests for comment.

Levine would like to see studies to determine whether inhibitors of the eIF4E-eIF4G interaction also overcome resistance to PI3K/protein kinase B (PKB; PKBA; AKT; AKT1)/mTOR (mammalian target of rapamycin; FRAP; RAFT1) inhibitors or synergize with existing drugs that target the pathway.

INSERM and the Gustave Roussy Institute have a pending patent covering the assessment of the status of the interaction of eIF4E with eIF4G in the eIF4F complex and with eIF4E binding protein 1 (eIF4EBP1) as markers for evaluating the sensitivity of cancer cells to a therapeutic treatment.

Lou, K.-J. SciBX 7(35); doi:10.1038/scibx.2014.1030

Published online Sept. 11, 2014

\section{REFERENCES}

1. Boussemart, L. et al. Nature; published online July 27, 2014; doi:10.1038/nature13572

Contact: Stéphan Vagner, Institut National de la Santé et de la Recherche Médicale (INSERM) UMR981, Villejuif, France e-mail: stephan.vagner@inserm.fr

Contact: Caroline Robert, same affiliation as above

e-mail: caroline.robert@gustaveroussy.fr

2. Konicek, B.W. et al. Cell Cycle 7, 2466-2471 (2008)

3. Silvera, D. et al. Nat. Rev. Cancer 10, 254-266 (2010)

4. Corcoran, R.B. et al. Oncotarget 2, 336-346 (2011)

5. Gupta, S.V. et al. AAPS J. 13, 357-364 (2011)

6. Liu, T. et al. J. Med. Chem. 55, 8859-8878 (2012)

7. Schatz, J.H. et al. J. Exp. Med. 208, 1799-1807 (2011)

8. Wolfe, A.L. et al. Nature; published online July 27,2014 doi:10.1038/nature13485

Contact: Hans-Guido Wendel, Memorial Sloan-Kettering Cancer Center, New York, N.Y.

e-mail: wendelh@mskcc.org

Contact: Gunnar Rätsch, same affiliation as above e-mail: ratschg@mskcc.org

9. Hong, D.S. et al. Clin. Cancer Res. 17, 6582-6591 (2011)

10. Kumari, S. et al. Nat. Chem. Biol. 3, 218-221 (2007)

11. Morris, M.J. et al. J. Am. Chem. Soc. 132, 17831-17839 (2010)

COMPANIES AND INSTITUTIONS MENTIONED

Chugai Pharmaceutical Co. Ltd. (Tokyo:4519), Tokyo, Japan

Daiichi Sankyo Co. Ltd. (Tokyo:4568), Tokyo, Japan

Egenix Inc., Millbrook, N.Y.

GlaxoSmithKline plc (LSE:GSK; NYSE:GSK), London, U.K.

Gustave Roussy Institute, Villejuif, France

Institut National de la Santé et de la Recherche Médicale, Villejuif, France

Isis Pharmaceuticals Inc. (NASDAQ:ISIS), Carlsbad, Calif. Memorial Sloan-Kettering Cancer Center, New York, N.Y. Roche (SIX:ROG; OTCQX:RHHBY), Basel, Switzerland University of Minnesota College of Pharmacy, Minneapolis, Minn.

University of Paris-Sud 11, Paris, France

University of Strasbourg, Strasbourg, France 